and lymphocytes within expansive aneurysms and co-localisation of NE with MMPs in AAA human samples.

This study suggests NE could be a regulator of aortic expansion and dissection, and therefore a potential target for AAA and TAD treatment. Further work is still needed to elucidate the causal mechanism by which NE takes its effect on AAA and TAD.

Conflict of interest no

\section{BS39 TRIB3 DEFICIENCY INCREASES PLAQUE STABILITY BY ALTERING MACROPHAGE FUNCTION}

'Laura Martinez Campesino*. 'University of Sheffield

10.1136/heartjnl-2019-BCS.201

Laura Martinez Campesino; Jessica M Johnston; Sheila E Francis; Endre Kiss-Toth; Heather L Wilson.

Department of Infection, Immunity \& Cardiovascular Disease, Medical School, Beech Hill Road,

University of Sheffield, UK.

Background Metabolic dysfunction and chronic inflammation under obesity are key factors promoting atherosclerosis. Macrophages are the major immune cell type found in atherosclerotic lesions, where plaque macrophages engulf oxidised lipids forming foam cells and also regulate plaque stability. Tribbles (Trib) 3 is a pseudokinase known to be involved in the regulation of adipose homeostasis, lipid and glucose metabolism and macrophage lipid uptake. We propose that Trib3 has a role in regulating metabolic and immune responses to obesity ultimately affecting atherosclerosis development.

Methodology. Trib3KO and Trib3WT mice were injected with rAAV8/mPCSK9 and fed with western diet for 12 weeks to promote atherosclerosis development. Atherosclerotic plaques were quantified using Oil-red-O and aortic root lesions were characterised histologically. Primary macrophages from bone marrow (BMDMs) were isolated from these strains and human monocyte derived macrophages (MDMs) were used to further characterise the effects of Trib3 regulating macrophage function.

Results En face analysis of the aortae showed no statistical differences in atheroma burden, while histological examination of the plaques indicated an increase in plaque stability in the absence of Trib3. Trib3KO BMDMs showed differential expression of genes involved in macrophage metabolism and regulation of the cytoskeleton. The differential expression of these genes was assessed in human MDMs following control or siTRIB3 treatment.

Conclusion These results suggest a role for Trib3 in the regulation of macrophage function promoting plaque stability, and may also have an impact on atherosclerosis development.

Conflict of interest No COI

\section{BS40 HYPOXIA INDUCES GENE-SPECIFIC EPIGENETIC MODIFICATIONS IN HUMAN CARDIAC FIBROBLASTS} ${ }^{1}$ Adam Russell-Hallinan*, ${ }^{2}$ Nadezhda Glezeva, ${ }^{2}$ Bruce Moran, ${ }^{3}$ Sudipto Das, ${ }^{2}$ John Baugh,
${ }^{1}$ Chris Watson. 'Queens University; ${ }^{2}$ University College Dublin; ${ }^{3}$ Royal College of Surgeons

10.1136/heartjnl-2019-BCS.202
Introduction Ischemia caused by coronary artery disease and myocardial infarction leads to aberrant ventricular remodelling and cardiac fibrosis. This occurs partly through accumulation of gene expression changes in resident fibroblasts, resulting in an overactive fibrotic phenotype. We have recently shown that human myocardial tissue hypoxia is associated with an enhanced pro-fibrotic gene profile in the tissue and, more significantly, that hypoxia-induced pro-fibrotic changes in cardiac fibroblasts are associated with global DNA hypermethylation.

Purpose Based on this epigenetics data, we have conducted a gene-specific methylation study to investigate methylation changes that occur in hypoxic ventricular fibroblasts and to gain novel insights into mechanisms that may contribute to post-ischemic cardiac remodelling.

Methods Human ventricular cardiac fibroblasts were exposed to $1 \%$ oxygen for up to 8 days. Global methylation changes were assessed using anti-5-methylcytosine $(5 \mathrm{MeC})$ staining, flow cytometry, QPCR, and western blot. Gene-specific methylation changes associated with hypoxia and an increased fibrotic state were determined by $5 \mathrm{MeC}$ immunoprecipitation and GeneChip human promoter arrays (Affymetrix), and validated by bisulphite genomic sequencing (BGS).

Results Hypoxia-induced pro-fibrotic changes in cardiac fibroblasts included increased cell proliferation and increased alpha smooth muscle actin, collagen 1, DNMT1 and DNMT3B expression which associated with global DNA hypermethylation. Array analysis revealed 37 gene-specific hypermethylation changes and 133-hypomethylation changes occurred in response to chronic hypoxia.

Conclusion Epigenetic modifications and changes in the epigenetic machinery identified in cardiac fibroblasts during prolonged hypoxia may contribute to the pro-fibrotic nature of the ischemic milieu in the heart during disease. The application of epigenetic-based therapy, such as DNA methylation modifiers, as a treatment option for cardiac pathologies associated with fibrosis and ischemia may provide therapeutic benefit.

Conflict of interest None

\section{BS41 ASYMMETRIC DIMETHYLARGININE INCREASES CALCIUM SENSING RECEPTOR SIGNALLING TO PROMOTE ENDOTHELIAL NO PRODUCTION}

${ }^{1}$ Laura Dowsett*, ${ }^{2}$ Lucie Duluc, ${ }^{1}$ Erin Higgins, ${ }^{1} J a m e s$ Leiper. 'Institute of Cardiovascular and Medical Sciences, The University of Glasgow; ${ }^{2} \mathrm{MRC}$ London Institute of Medical Research, Imperial College London

\subsection{6/heartjnl-2019-BCS.203}

Objective The post-translational methylation of arginine residues by PRMT enzymes can yield asymmetric dimethylarginine (ADMA); which, following proteolysis is released into the cytosol and enters the circulation. Elevated plasma ADMA concentrations are recognised as a risk factor for cardiovascular disease including hypertension, atherosclerosis and stroke. In part, this is due to ADMA acting as a competitive inhibitor of the NOS enzymes reducing NO production and contributing to endothelial dysfunction. However, recently we have identified the Calcium sensing receptor (CaSR) as a target for physiological concentrations of ADMA. CaSR is a GPCR which binds both $\mathrm{Ca} 2+$ and amino acids, and has been shown to positively modulate $\mathrm{NO}$ production in endothelial cells 
(ECs). Our objective was to determine whether physiological concentrations of ADMA had a positive effect on NO production via CaSR stimulation.

Methods Inducible CaSR overexpressing HEK293 cells (HekCaSR) were generated using the FLP-IN T-REX transfection method. Intracellular $\mathrm{Ca} 2+$ mobilisation was assessed using the $\mathrm{Ca} 2+$ sensitive dye CAL520, while cAMP accumulation was measured using an ELISA kit. CaSR was stimulated by $\mathrm{Gd} 3+$ in the presence of $10 \mu \mathrm{M}$ ADMA. ADMA-CaSR signalling in ECs was investigated using human lung microvascular ECs (HuLMVECs). NO production was assessed using the fluorescent sensor DAF-AM. The CaSR antagonist NPS-2143 was used to show specificity to CaSR signalling.

Results $\mathrm{Gd} 3+$ releases stored intracellular $\mathrm{Ca} 2+$ and suppresses cAMP production in a dose dependent manner in HEK-CaSR cells. Incubation with $10 \mu \mathrm{M}$ ADMA for 1 hour prior to $\mathrm{Gd} 3$ + stimulation was found to sensitise CaSR and shift the dose-response curve for $\mathrm{Ca} 2+$ mobilisation leftward (CTRL EC50 0.16mM, ADMA EC50 $0.05 \mathrm{mM}$ p=0.01). ADMA pre-treatment suppressed forskolin induced cAMP production to $70 \%$ of the maximal response. $\mathrm{Ca} 2+$ stimulation of HuLMVECs releases intracellular $\mathrm{Ca} 2+$ in a dose dependent manner, in the presence of $10 \mu \mathrm{M}$ ADMA maximal fluorescence is increased with no change to the EC50. Given cytosolic $\mathrm{Ca} 2+$ release increases NOS activity we determined whether low concentrations of ADMA $(10 \mu \mathrm{M})$ promoted NO production. At maximal CaSR stimulation ADMA increased NO production by $40 \%(\mathrm{p}=0.04)$. Consistent with previous reports higher concentrations of ADMA $(100 \mu \mathrm{M})$ blocked NO production through inhibition of NOS. The action of
ADMA was blocked by the addition of the CaSR inhibitor NPS-2143.

Conclusion Physiological concentrations of ADMA increase CaSR-mediated intracellular $\mathrm{Ca} 2+$ release and increase CaSRmediated suppression of cAMP synthesis. In ECs, physiological concentrations of ADMA increase $\mathrm{Ca} 2+$ stimulated $\mathrm{NO}$ synthesis. In contrast pathophysiological concentrations directly inhibit EC NO synthase. These data suggest that physiological concentrations of ADMA positively regulate vascular reactivity while high concentrations of ADMA seen in cardiovascular disease reduce vascular function through NO blockade.

Conflict of interest None

\section{BS42 HOW WARFARIN AND ANTIPLATELETS AFFECT CLOT STRUCTURE IN ATRIAL FIBRILLATION}

${ }^{1}$ Ahsan Khan* ${ }^{2}{ }^{2}$ Eduard Shantsila, ${ }^{2}$ Y.C. Lau, ${ }^{3}$ Lewis Hardy, ${ }^{3}$ Helen Philippou, ${ }^{1}$ Gregory Lip. ${ }^{1}$ Liverpool Heart \& Chest Hospital NHS Foundation Trust; ${ }^{2}$ University of Birmingham; ${ }^{3}$ University of Leeds

\subsection{6/heartjnl-2019-BCS.204}

Introduction Atrial fibrillation (AF) and coronary artery disease (CAD) are two common cardiovascular conditions associated with increased morbidity and mortality. Both conditions bring a risk of thrombosis and thus antithrombotic therapy is indicated. Despite recognised treatment, stroke and myocardial infarctions still occur. Accordingly, the identification of patients whose haemostasis remains unimpaired by treatment is valuable.

Abstract BS42 Table 1 Demographic, clinical and heamostatic characteristic of patients with atrial fibrillation and coronary artery disease (control group)

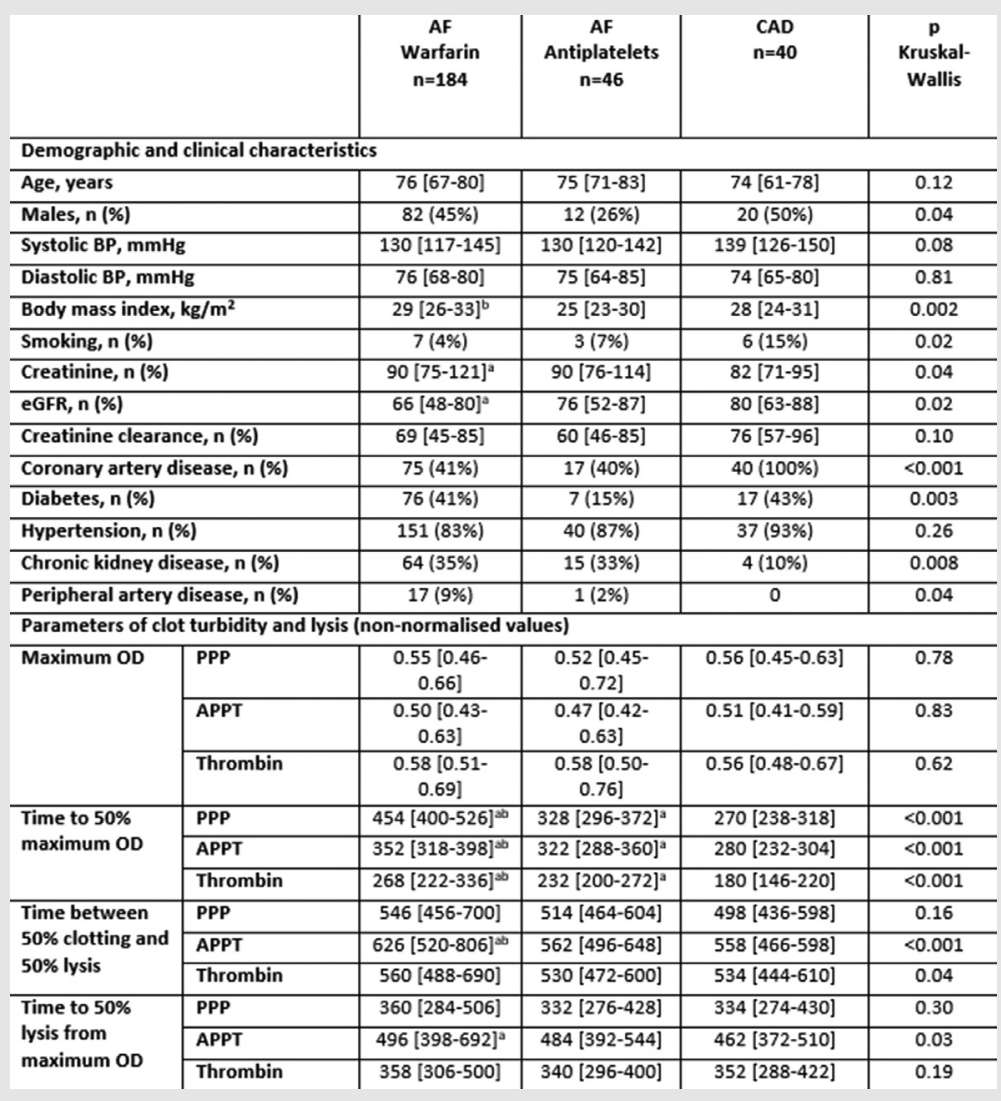

